\title{
Erratum: Comprehensive Studies of Solar Activity on the CORONAS-F Satellite* [Astronomy Letters 28, 401-410 (2002)]
}

\author{
V. N. Oraevsky ${ }^{1}$ and I. I. Sobelman ${ }^{2}$ \\ ${ }^{1}$ Institute of Terrestrial Magnetism, Ionosphere, and Radio-Wave Propagation, Russian Academy of Sciences, \\ Troitsk, Moscow oblast, 142090 Russia; E-mail: oraevsky@izmiran.rssi.ru \\ ${ }^{2}$ Lebedev Physical Institute, Russian Academy of Sciences, Leninskǐ pr. 53, Moscow, 117924 Russia \\ Received December 27, 2001
}

\begin{abstract}
The first results of comprehensive CORONAS-F observations of solar activity are presented. The CORONAS-F instrumentation and principal scientific objectives are briefly described and examples of the first results of data reduction are given. (c) 2002 MAIK “Nauka/Interperiodica”.
\end{abstract}

Key words: Sun; astronomical observing techniques, equipment and instruments

THE CORONAS PROGRAM

AND THE CORONAS-F PROJECT

The CORONAS (Comprehensive Orbital NearEarth Solar Activity Observations) International Program, as part of which the CORONAS-F nearEarth spaceborne solar observatory was launched, is intended for studies of the Sun at various phases of the 11 -year solar cycle. On the previous CORONASI satellite (launched in 1994), the Sun was observed near the minimum of its activity. The CORONAS-F will study the solar activity near the maximum of the current cycle 23. On July 31, 2001, CORONAS-F was placed in an orbit with the following parameters: an orbital inclination of $82.49^{\circ}$, a minimum altitude of $500.9 \mathrm{~km}$, a maximum altitude of $548.5 \mathrm{~km}$, and a revolution period of 94.859 min. Such an orbit provides repetitive $\sim 20$ day-long periods of continuous solar observations, which is of particular importance in helioseismology and in patrolling solar flares. The actually achieved spacecraft attitude stabilization turned out to be a factor of 3 to 5 better than the projected one (a few arcseconds per second), which allows the spatial resolution of solar observations to be appreciably improved.

\section{SCIENTIFIC OBJECTIVES}

\section{OF THE CORONAS-F PROJECT}

The principal scientific objectives of the CORONAS-F project are observations of global solar oscillations, investigation of the seismology of the solar

${ }^{*}$ This article was republished due to two color figures (nos. 4 and 5) missed in the original translation. Our apologies to the authors and the readers of the journal. interior and internal structure, comprehensive studies of powerful dynamical processes on the active Sun (active regions, flares, plasma ejections) over a wide wavelength range, from optical to gamma rays, including the radiation from solar cosmic-ray particles accelerated during active solar events, conditions for their escape, propagation in the interplanetary magnetic field, and impact on the Earth's magnetosphere.

\section{SCIENTIFIC INSTRUMENTATION AND THE FIRST OBSERVATIONAL RESULTS}

In accordance with the CORONAS-F objectives, the scientific instrumentation of the spacecraft includes four major groups of instruments: an instrument to detect global solar oscillations; X-ray instruments to image active regions on the Sun with a spatial resolution of $\sim 2^{\prime \prime}-3^{\prime \prime}$ instruments to measure the fluxes of electromagnetic radiation from active

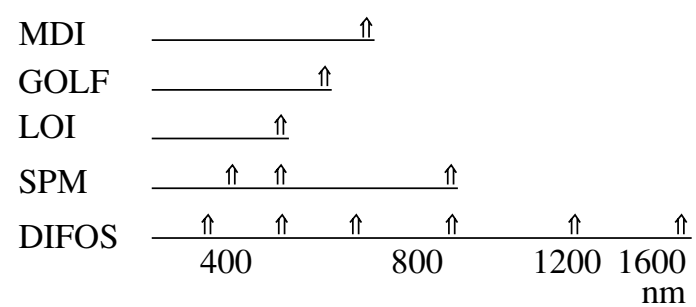

Fig. 1. Comparison of the DIFOS (CORONAS-F) channels with the SOHO instruments (MDIMichelson Doppler Imaging of solar oscillations, GOLF-Global low-degree velocity, LOI-Luminosity Oscillation Imager, SPM-SunPhotoMeter). 
Table 1. The CORONAS-F instruments

\begin{tabular}{|c|c|c|c|}
\hline Instrument & Purpose & Designer & $\begin{array}{l}\text { Principal } \\
\text { investigators }\end{array}$ \\
\hline \multicolumn{4}{|c|}{ Helioseismology } \\
\hline DIFOS spectrophotometer & Helioseismological monitoring & IZMIRAN & V.N. Oraevsky \\
\hline \multicolumn{4}{|c|}{ High-angular-resolution monochromatic imaging } \\
\hline $\begin{array}{l}\text { SRT-C X-ray } \\
\text { telescope }\end{array}$ & $\begin{array}{l}\text { Studying the spatial structure and dynamics } \\
\text { of the upper solar atmosphere using } \\
\text { narrow-band XUV images }\end{array}$ & FIAN & $\begin{array}{l}\text { I.I. Sobelman } \\
\text { I.A. Zhitnik }\end{array}$ \\
\hline $\begin{array}{l}\text { RES-C X-ray } \\
\text { spectroheliograph }\end{array}$ & $\begin{array}{l}\text { Diagnosing the hot solar atmospheric } \\
\text { plasma using images in X and XUV spectral } \\
\text { lines }\end{array}$ & FIAN & $\begin{array}{l}\text { I.I. Sobelman } \\
\text { I.A. Zhitnik }\end{array}$ \\
\hline $\begin{array}{l}\text { DIOGENESS } \\
\text { spectrophotometer }\end{array}$ & $\begin{array}{l}\text { Studying the } X \text {-ray radiation from solar } \\
\text { active regions and flares }\end{array}$ & SRC PAS* & J. Silvestr \\
\hline \multicolumn{4}{|c|}{ Measuring the fluxes and polarization of electromagnetic radiation (from UV to $\gamma$ rays) } \\
\hline $\begin{array}{l}\text { RESIK X-ray } \\
\text { spectrometer }\end{array}$ & $\begin{array}{l}\text { Studying the solar X-ray radiation with a } \\
\text { high spectral resolution }\end{array}$ & SRC PAS* & J. Silvestr \\
\hline $\begin{array}{l}\text { SPR-N solar } \\
\text { spectropolarimeter }\end{array}$ & $\begin{array}{l}\text { Studying the polarization of } \mathrm{X} \text {-ray radiation } \\
\text { from solar flares }\end{array}$ & $\begin{array}{l}\text { FIAN } \\
\text { NIIYaF }\end{array}$ & $\begin{array}{l}\text { I.I. Sobelman } \\
\text { I.P. Tindo } \\
\text { S.I. Svertilov }\end{array}$ \\
\hline IRIS flare spectrometer & Studying the X-ray solar flare activity & FTI & G.E. Kocharov \\
\hline $\begin{array}{l}\text { GELIKON gamma-ray } \\
\text { spectrometer }\end{array}$ & $\begin{array}{l}\text { Studying the } X \text {-ray and gamma-ray solar } \\
\text { flare activity }\end{array}$ & FTI & E.P. Masetz \\
\hline RPS X-ray spectrometer & $\begin{array}{l}\text { Studying the X-ray radiation from solar } \\
\text { flares and their precursors }\end{array}$ & $\begin{array}{l}\text { IKI RAS } \\
\text { MIPhI }\end{array}$ & $\begin{array}{l}\text { V.M. Pankov } \\
\text { Yu.D. Kotov }\end{array}$ \\
\hline $\begin{array}{l}\text { ATS amplitude-time } \\
\text { spectrometer }\end{array}$ & $\begin{array}{l}\text { Studying the } X \text {-ray and gamma-ray } \\
\text { radiation from solar flares }\end{array}$ & MIPhI & Yu.D. Kotov \\
\hline $\begin{array}{l}\text { SUVR-Sp-C solar } \\
\text { ultraviolet radiometer }\end{array}$ & Studying the variations in total UV solar flux & IPG & T.V. Kazachevskaya \\
\hline $\begin{array}{l}\text { VUSS-L ultraviolet solar } \\
\text { spectrophotometer }\end{array}$ & $\begin{array}{l}\text { Studying the solar UV radiation near the } L_{\alpha} \\
\text { resonance line }\end{array}$ & IPG & A.A. Nusinov \\
\hline \multicolumn{4}{|c|}{ Studying solar corpuscular fluxes } \\
\hline $\begin{array}{l}\text { Instrumentation to study } \\
\text { SCRs }\end{array}$ & Studying solar cosmic rays & $\begin{array}{l}\text { NIIYaPh } \\
\text { MGU }\end{array}$ & S.N. Kuznetsov \\
\hline \multicolumn{4}{|c|}{ Gathering and studying of scientific information } \\
\hline $\begin{array}{l}\text { Scientific Information } \\
\text { Acquisition System } \\
\text { (SSNI) }\end{array}$ & $\begin{array}{l}\text { Controlling the scientific instrumentation } \\
\text { and the modes of its operation }\end{array}$ & IZMIRAN & $\begin{array}{l}\text { V.N. Oraevsky } \\
\text { A.I. Stepanov }\end{array}$ \\
\hline
\end{tabular}

* Space Research Center of the Polish Academy of Sciences. 


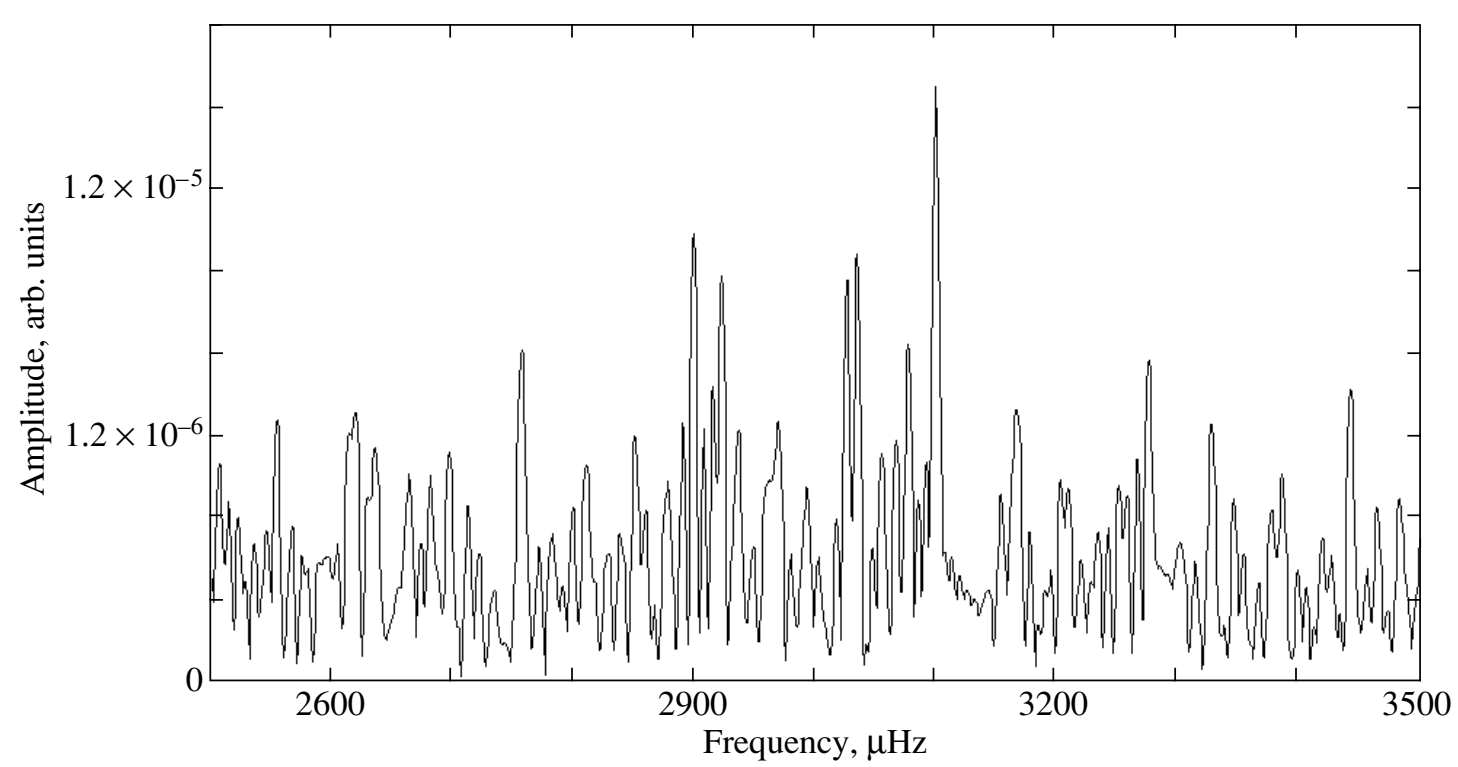

Fig. 2. The relative amplitudes of 5-min solar oscillation modes at a wavelength of $350 \mathrm{~nm}$ as inferred from the results of the DIFOS data reduction.

regions and flares; and instruments to study solar corpuscular fluxes. The wide range of measurements of the electromagnetic spectrum and solar cosmic-ray particle [both neutral (neutrons) and charged (electrons, protons, nuclei)] fluxes yields the fullest picture of the physical processes in active regions on the Sun. The CORONAS-F instruments and their main purpose are given in Table 1.

The main operational control of the scientific instrumentation is performed from the Flight Control Center located at the Cosmic Information Technology Center, Institute of Terrestrial Magnetism, Ionosphere, and Radio-Wave Propagation (IZMIRAN), Troitsk. Commands are sent to the spacecraft on a daily basis. Up to 24 kbytes of commands are sent simultaneously. Apart from controlling the modes of operation, the controllers of the scientific instruments are dynamically reprogrammed in flight, which allows

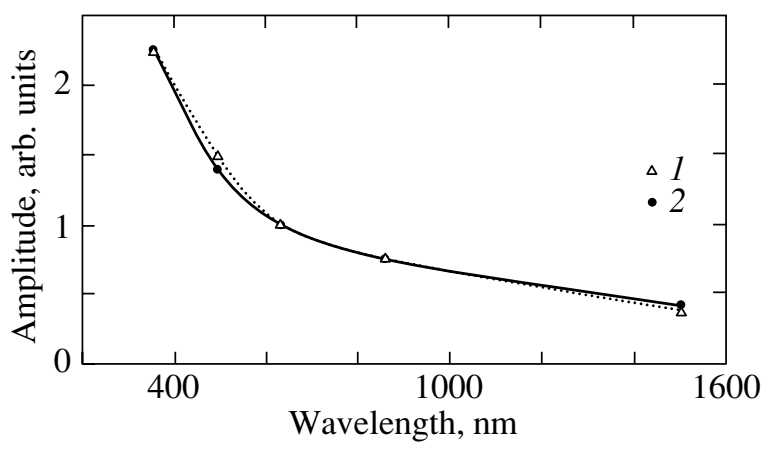

Fig. 3. The relative amplitude of free solar oscillations versus wavelength. their software to be customized for current observations. Requests for control through the Flight Control Center of the Cosmic Information Technology Center (IZMIRAN) are collected via E-mail with no less than 10 min before the control session, which ensures the required speed and flexibility in controlling the scientific instrumetation.

\section{THE DIFOS SPECTROPHOTOMETER}

The DIFOS spectrophotometer is designed to measure fluctuations in the intensity of solar optical radiation in an effort to obtain the spectrum of free oscillations. The intensity is simultaneously measured in six optical spectral bands: 350, 500, 650, 850,1100 , and $1500 \mathrm{~nm}$ with a band width equal to $10 \%$ of the central frequency. In comparison with the CORONAS-I project (Lebedev et al. 1995), the photometer was significantly upgraded: the detector sensitivity increased by more than an order of magnitude and the spectral range of observations was extended by almost a factor of 2 ; concurrently, the number of spectral channels increased from three to six. Global solar oscillations are being observed over a wide spectral range, including the most informative UV line. The intensity of the radiation from global solar oscillations in this line appreciably exceeds the radiation intensity in other lines (Oraevsky et al. 1999). Figure 1 compares the DIFOS channels with the SOHO instruments (1988).

Figure 2 shows the relative amplitudes of 5 -min solar oscillation modes at a wavelength of $350 \mathrm{~nm}$ during November 28-30, 2001 as inferred from the 
Table 2. Characteristics of the SRT-C telescope

\begin{tabular}{|c|c|c|c|c|c|}
\hline Channel & $\begin{array}{c}\text { Spectral range, } \\
\AA\end{array}$ & Ion lines & $\begin{array}{l}\text { Field of } \\
\text { view, } \\
\text { arcmin }\end{array}$ & $\begin{array}{l}\text { Angular } \\
\text { scale } \\
\text { per cell }\end{array}$ & Mode of operation \\
\hline \multirow[t]{4}{*}{ BP1 } & 171 & Fe IX-Fe X & $42 \times 48$ & $2^{\prime \prime} .5$ & Full disk \\
\hline & 195 & Fe XII & & & \\
\hline & 284 & Fe XV & & & \\
\hline & 304 & He II & & & \\
\hline \multirow[t]{2}{*}{ BP2 } & 175 & Fe IX-Fe XI & $45 \times 50$ & $2^{\prime \prime} .6$ & Full disk, pointing at corona \\
\hline & 304 & $\mathrm{HeII}+\mathrm{SiXI}$ & $45 \times 50$ & $2^{\prime \prime} 6$ & up to several solal radu \\
\hline $\begin{array}{l}\text { Optical sensors OS1 } \\
\text { and OS2 }\end{array}$ & $4000-8000$ & & Circular $11^{\circ}$ & $1^{\prime} \times 2^{\prime}$ & Stars $>12^{m}$ \\
\hline
\end{tabular}

results of the DIFOS data reduction. Similar results were obtained for the other channels. After averaging over the modes, the mean relative amplitudes were determined for each of the six channels and plotted against wavelength (see Fig. 3). All data points fall on a smooth curve fitted by a $\lambda^{-1.2}$ law (1). A similar result was established for another interval of observations, November 30-December 3, 2001 (2). A comparison of the DIFOS result with the results in the range of ground-based observations (Jimenez et al. 1998) gives close agreement. According to ground-based measurements, the amplitude ratio of global oscillations at wavelengths of 500 and $680 \mathrm{~nm}$ was 1.6, while for the DIFOS measurements, the amplitude ratio at 500 and $650 \mathrm{~nm}$ was 1.5. For a different pair of wavelengths, the amplitude ratio at 500 and $870 \mathrm{~nm}$ was 2.2 for ground-based observations, while the amplitude ratio at 500 and $850 \mathrm{~nm}$ for the DIFOS data was 2.

THE SPIRIT (SRT-RES-SPR)

ASTROPHYSICAL COMPLEX TO STUDY THE SOLAR X-RAY RADIATION

The SPIRIT astrophysical complex consists of the SRT-C solar X-ray telescope, the RES-C X-ray spectroheliograph, and the SPR-N spectropolarimeter. It is designed to solve a wide variety of problems in solar physics based on XUV imaging spectroscopy of the Sun (soft X-ray and extreme UV solar spectroscopy). This method consists in obtaining simultaneously solar images and spectra with high angular and spectral resolutions in narrow portions of the XUV range that characterize the various temperature layers of solar plasma. It was applied in the CORONAS-I project, in which the TEREK-C multichannel telescope and the RES-C spectroheliometer in the wavelength range $0.18-30.4 \mathrm{~nm}$ designed at the Lebedev Physical Institute (Russian Academy of Science) were used (Sobelman et al. 1996; Zhitnik et al. 1998).

Observations with the SPIRIT astrophysical complex onboard the CORONAS-F satellite will allow the following processes to be investigated: the formation and development of solar flares from temporal variations and changes in the structure, spectra, and polarization of the flare regions; nonstationary phenomena in solar plasma (hot points, coronal mass ejections, and others) - the frequency of their occurrence, conditions for their emergence, dynamics of their development, and residual phenomena; and the helium distribution in the transition layer and in the near and far coronas. They also allow us to search for the possible sources of the solar wind, to study the processes of its generation, to elucidate the relationships of the observed solar-wind parameters to solar processes, and to diagnose the structural elements of active regions, areas of the quiet Sun, and coronal holes on time scales from $0.1 \mathrm{~s}$ to tens of days.

The SPIRIT complex is distinguished by a wide spectral range $(0.04-100 \mathrm{keV})$ and by high spatial (up to $3^{\prime \prime}$ ), spectral (up to $2 \times 10^{3}$ ), and temporal $(0.01 \mathrm{~s})$ resolutions intended for observations near maximum solar activity. The SRT-C telescope is a new instrument with significantly improved characteristics compared to the TEREK-C telescope that successfully operated on the CORONAS-I satellite. The working aperture of the optical channels increased from 30 to $100 \mathrm{~mm}$ with the simultaneous increase in actual angular resolution. The number of spectral subbands also increased. The RES-C$F$ spectroheliometer is a modification of the RESC-I spectroheliometer that successfully operated on the CORONAS-I satellite with an extended spectral range and improved characteristics. In the SRT-C 
Table 3. Characteristics of the RES-C X-ray spectroheliograph

\begin{tabular}{|c|c|c|c|c|c|}
\hline Channel & $\begin{array}{c}\text { Spectral range, } \\
\AA\end{array}$ & Ion lines & $\begin{array}{c}\text { Spectral resolution, } \\
\AA \text { per cell }\end{array}$ & $\begin{array}{l}\text { Angular } \\
\text { scale } \\
\text { per cell }\end{array}$ & Mode of operation \\
\hline Fe XXV & $1.85-1.87$ & $\begin{array}{l}\text { Fe XXIV, } \\
\text { FeXXV }\end{array}$ & $1.5 \times 10^{-4}$ & $1^{\prime}$ & $\begin{array}{l}\text { Image and } \\
\text { spectrum from the } \\
\text { entire disk }\end{array}$ \\
\hline $\begin{array}{l}\text { Mg XII (two crossed } \\
\text { subchannels) }\end{array}$ & $8.41-8.43$ & Mg XII doublet & $3 \times 10^{-3}$ & $4.1^{\prime \prime}$ & Same \\
\hline $\begin{array}{l}\text { XUV (two crossed } \\
\text { subchannels) }\end{array}$ & $285-335$ & $\begin{array}{l}\text { Fe VIII, X, } \\
\text { XI } \\
\text { XII, XIII, } \\
\text { XXIV, } \\
\text { O VI S IX } \\
\text { He II, Si XI }\end{array}$ & 0.03 & $\begin{array}{l}6^{\prime \prime} \text { (perpen- } \\
\text { dicular to } \\
\text { dispersion) }\end{array}$ & $\begin{array}{l}\text { Spectral images of } \\
\text { the disk in all lines }\end{array}$ \\
\hline
\end{tabular}

and RES-C-F instruments, the resolution and sensitivity of the imaging detectors were increased, their dynamical range was expanded, and a powerful highperformance computer with a large amount of random access memory was used in the electronic system. The new instruments provide an increase in temporal resolution and sensitivity by up to a factor of $10 \mathrm{com}-$ pared to the TEREK and RES instruments on the CORONAS-I satellite and by a factor of 3 compared to the EIT/SOHO telescope.

\section{The SRT-C Solar X-ray Telescope}

The SRT-C solar X-ray telescope is designed for $\mathrm{X}$-ray imaging of the Sun with a high spatial resolution. It includes XUV channels, BP1 and BP2, and two optical sensors for the telescope attitude control using stars. The telescope characteristics are listed in Table 2.

$\mathrm{X}$-ray mirrors with multilayer Mo-Si coating are used for imaging in the $\mathrm{X}$-ray channels of the instrument: in channel BP1, a Ritchey-Chretien objective with a diameter of $120 \mathrm{~mm}$ and a focal length of $1660 \mathrm{~mm}$ that has four sectors with coverages at wavelengths of $171,195,284$, and $304 \AA$ (an ana$\log$ of the EIT/SOHO telescope objective); and in channel BP2, off-axis paraboloids with a diameter of $100 \mathrm{~mm}$ and a focal length of $1500 \mathrm{~mm}$.

The imaging detectors in all channels are CCD arrays; image intensifiers, which also act as electronic shutters, are used in the X-ray channels. The brightness dynamic range in the X-ray channels is of the order of $10^{5}$. The exposure times can vary between 0.01 and $600 \mathrm{~s}$.

The mirrors of channel BP2 are equipped with angular alignment and focusing systems to correct for possible changes in alignment when placing the satellite in orbit. In addition, the channel is supplied with artificial moons blocked by commands and with additional optical windows, which in combination with mirror deflection makes it possible to observe portions of the $\mathrm{X}$-ray corona within several solar radii.

\section{The RES-C X-ray Spectroheliograph}

The RES-C spectroheliograph is designed to image the Sun in spectral lines of highly ionized atoms of abundant elements (helium, magnesium, iron, silicon) with a high spatial resolution and to measure the linear polarization in spectral lines. The instrument includes three XUV channels: Fe, Mg, and XUV. The RES-C characteristics are listed in Table 3.

$\mathrm{X}$-ray optics is used in the RES-C instrument: in the Fe XXV channel, a ring Bragg mirror made of crystalline quartz with a sphere radius of $196.6 \mathrm{~mm}$ operating at a Bragg angle of $52^{\circ}$; in the $\mathrm{Mg}$ XII channel, two spherical Bragg mirrors made of crystalline quartz with a sphere radius of $1286 \mathrm{~mm}$ operating at angle $81.7^{\circ}$; in the XUV channels, two holographic diffraction gratings with a period of 3600lines per $\mathrm{mm}$ whose spectra are focused on the detectors using multilayer Mo-Si-coated mirrors with a radius of $1600 \mathrm{~mm}$ were used: spherical and parabolic ones for the wavelength ranges 180-205 $\AA$ and 285$335 \AA$, respectively.

The imaging detectors in the XUV channels, just as those in the SRT-C instrument, are CCD arrays in combination with image intensifiers. The dynamic range is of the order of $10^{5}$ and the exposure time ranges from 0.01 to $600 \mathrm{~s}$. In the $\mathrm{Mg}$ XII and Fe XXV channels, $\mathrm{X}$-ray radiation is recorded by the open CCD arrays. 

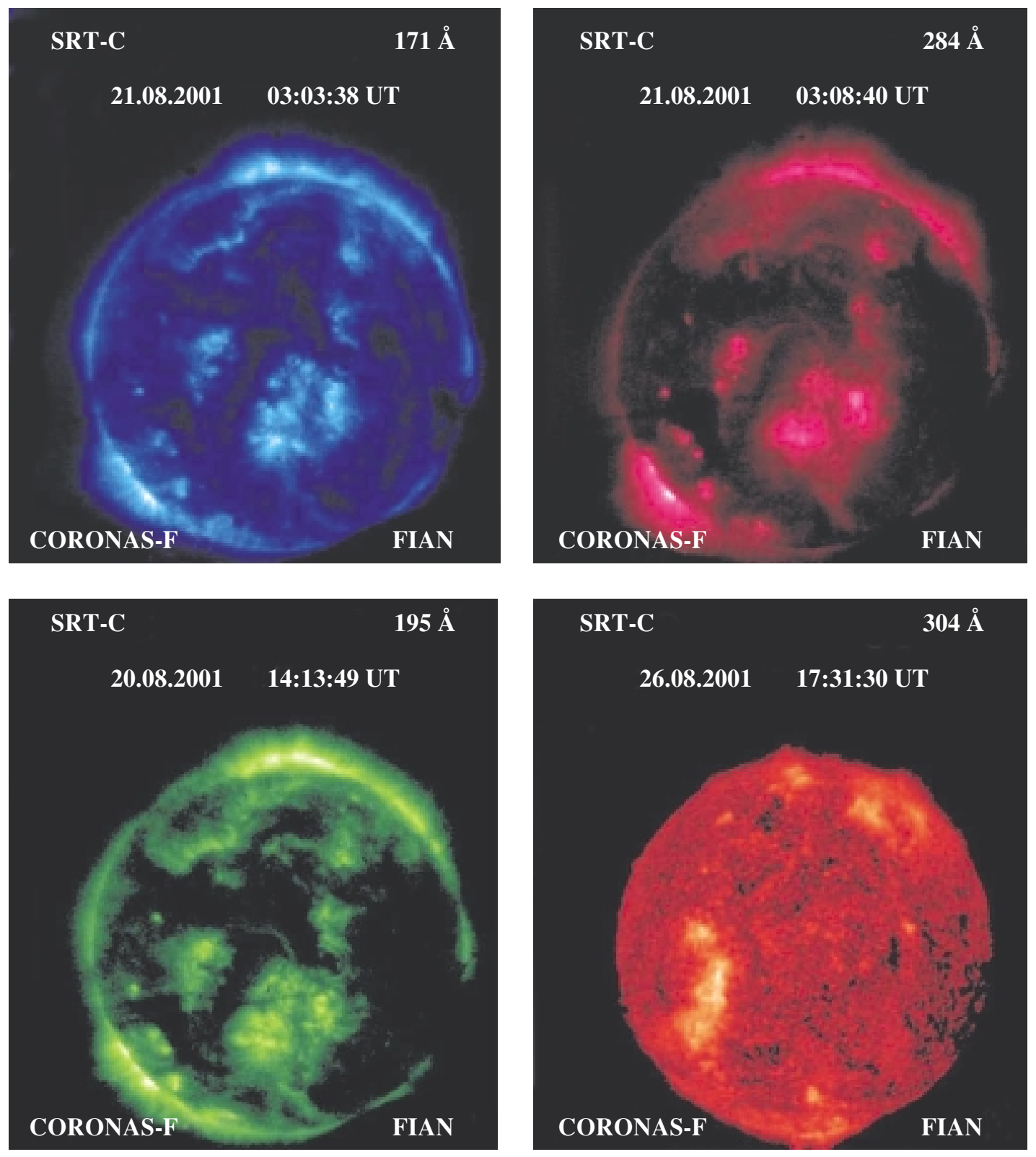

Fig. 4. The solar images at wavelengths of 171, 195, 284, and $304 \AA$ Abtained in channel BP1 of the SRT-C instrument. 


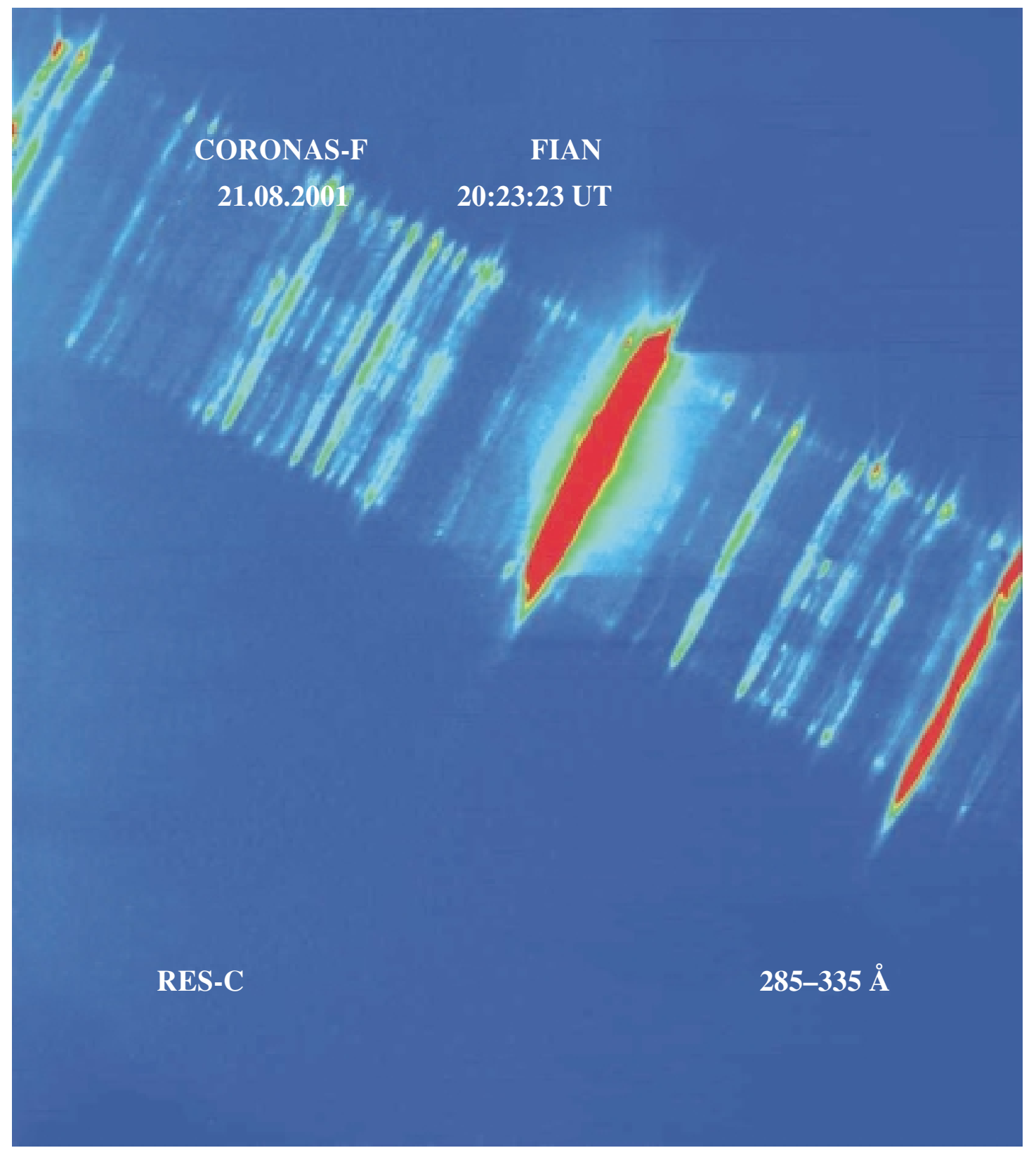

Fig. 5. The spectroheliogram in the range 285-335 A taken in the XUV channel of the RES-C instrument. 


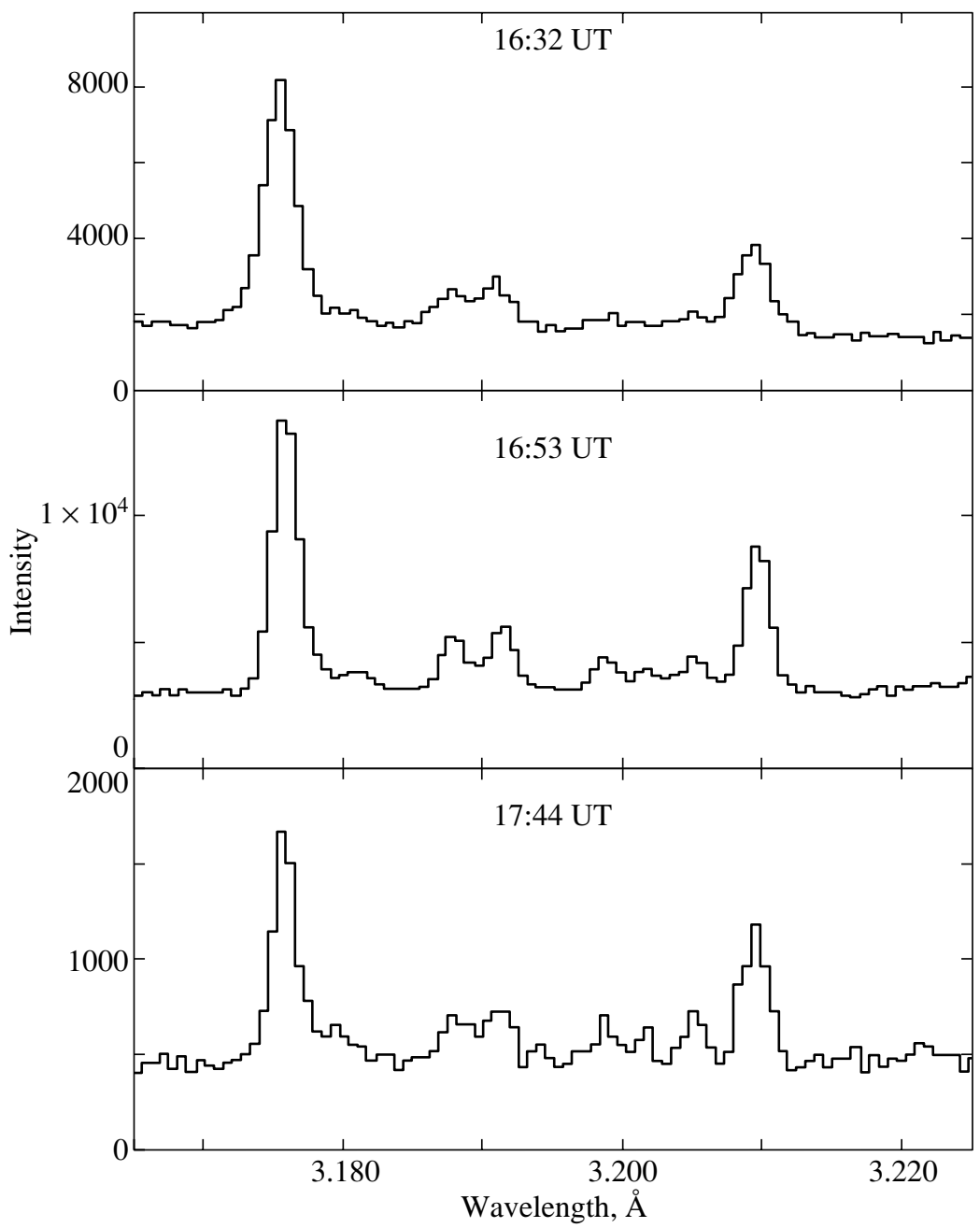

Fig. 6. Ca XIX line spectra with a high spatial resolution $\left(\sim 5^{\prime \prime}\right)$ at the growth phase, intermediate phase, and the decay phase of the strongest flare in the current solar cycle (August 25, 2001).

\section{The SPR-N Solar Spectropolarimeter}

The SPR-N solar X-ray spectropolarimeter measures the polarization of continuum $\mathrm{X}$-ray radiation from solar flares in the spectral range $20-100 \mathrm{keV}$ (nonthermal radiation from the accelerated electrons generated in flares). The instrument has the following characteristics:

$\begin{array}{ll}\text { Spectral range } & 20-100 \mathrm{keV} \\ \text { Subbands } & 20-40 \mathrm{keV} \\ & 40-60 \mathrm{keV} \\ & 60-100 \mathrm{keV} \\ \text { Polarization detection sensitivity } & \sim 5 \% \text { at flux } \\ & 10^{-6} \mathrm{erg} \mathrm{cm}^{-2} \mathrm{~s}^{-1} \\ \text { Temporal resolution } & 4-16 \mathrm{~s}\end{array}$

The SPR-N instrument has two detection chan- nels: a polarization detector and a patrol detector. The polarization detector contains an X-ray radiation scatterer (a hexahedral Be prism) and a system of three pairs of scintillation detectors (SSD), which measure the intensity of the scattered radiation. CsI(Na) crystal scintillators with photomultipliers are used to record X-ray radiation. To eliminate the systematic errors during polarization measurements attributable to the possible sensitivity drift in individual channels, the polarization detector was mounted on a turning drive. For typical solar flares of importance I, II, and III, the expected sensitivity of polarization measurement is $\sim 5 \%$ at a flux of $\sim 10^{6}$ phot. $\mathrm{cm}^{-2} \mathrm{~s}^{-1}(E=20-100 \mathrm{keV})$ and an integration time of $\sim 8 \mathrm{~s}$. The patrol detector is designed to measure the total intensity of the $\mathrm{X}$-ray radiation from solar flares at energies $20-100 \mathrm{keV}$. It consists of a $\operatorname{CsI}(\mathrm{Na})$ crystal placed behind an anticoincidence shield and a photomultiplier.

Apart from the general scientific program of the 


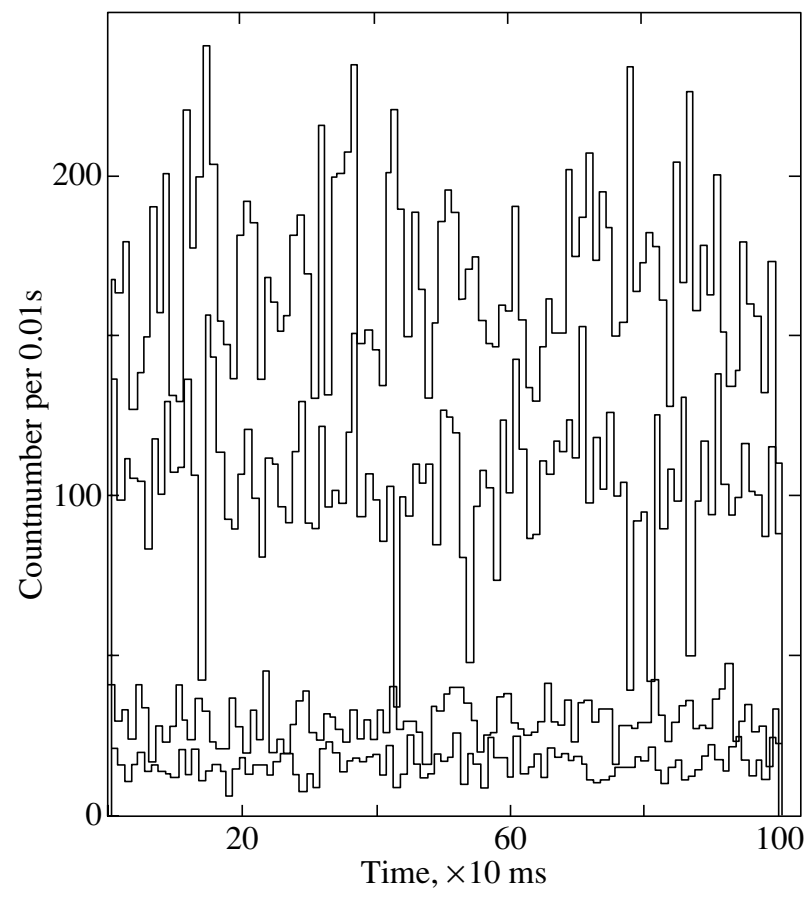

Fig. 7. An intensity record of hard X-ray radiation on September 27, 2001, 18:26 UT in the energy range 20-100 keV with a high temporal resolution in four energy channels (from top to bottom: 20-25, 25-35, 35-100, and 100-200 keV).

CORONAS-F project, the programs of joint observations with instruments on other spacecraft that will operate simultaneously with CORONAS-F, primarily with instruments on the SOHO international spaceborne observatory, the TRACE telescope, YOHKOH, and others, are envisaged. It is planned that the CORONAS-F observations will be coordinated with observations at ground-based observatories, in particular, with the Institute of Solar-Terrestrial Physics (Itkutsk), the Crimean Astrophysical Observatory, and other ground-based observatories that carry out solar observations.

Test experiments and preliminary scientific results obtained during the first three months of observations show that the entire instrumentation of the SRT-RES-SPR operates normally. Figure 4 shows the solar images at $171,195,284$, and $304 \AA$ obtained in channel BP1 of the SRT-C instrument. Figure 5 shows the spectroheliogram in the range 285-335 $\AA$ taken in the XUV channel of the RES$\mathrm{C}$ instrument.

\section{THE DIOGENESS SPECTROPHOTOMETER AND THE RESIK X-RAY SPECTROMETER}

The RESIK and DIOGENESS instruments measure the solar spectra in the wavelength range $3-7 \AA$. They are designed to investigate the $\mathrm{X}$-ray radiation from active regions and flares with a high spectral resolution. Their solar X-ray spectra are comparable in spectral and temporal resolution to the best observations performed to date. Because of the CORONAS$\mathrm{F}$ polar orbit and the complete coverage of a wide spectral range, they are a valuable complement to the Yohkoh spectra. Since the detectors of the RESIK instrument and the Yohkoh spectrometers are saturated at high X-ray fluxes, the DIOGENESS instrument is currently the world's only operating spectrometer that takes the spectra of intense flares of importance above M2 (as exemplified by the X5.3 flare on August 25, 2001).

Figure 6 shows Ca XIX line spectra with a high spatial resolution $\left(\sim 5^{\prime \prime}\right)$ at the growth phase, intermediate phase, and the decay phase of the strongest flare in the current solar cycle (August 25, 2001). The temperature of the emitting plasma decreases from $2.5 \times 10^{6} \mathrm{~K}$ at the growth phase to $1.2 \times 10^{6} \mathrm{~K}$ at the decay phase. The spectra exhibit changes in the line width, which points to the role of plasma turbulence during the flare evolution. A detailed analysis of the relative intensities will allow one to study the energy balance in flares and the role of non-Maxwellian and nonequilibrium processes in the flare energy release region. In the spectral range in question $(3-7 \AA)$, such spectra for an X flare were obtained with such a high spatial resolution for the first time. They provide information on the many emission lines produced by the collisional excitation of atoms, the excitation of inner atomic shells, and dielecton recombination. 


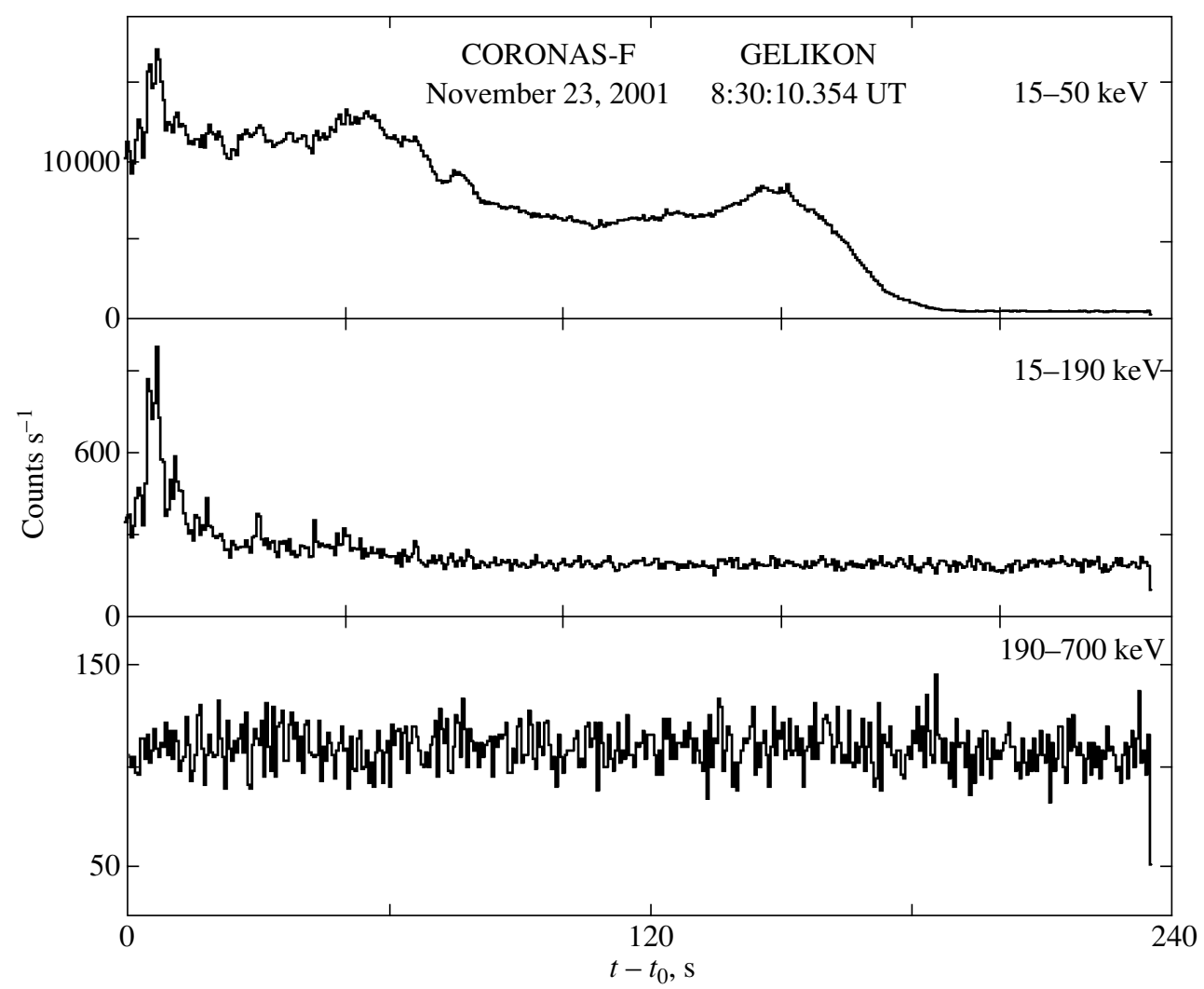

Fig. 8. The time profile for the intense solar flare of November 23, 2001, in three energy bands with a high temporal resolution, from 2 to $256 \mathrm{~ms}$.

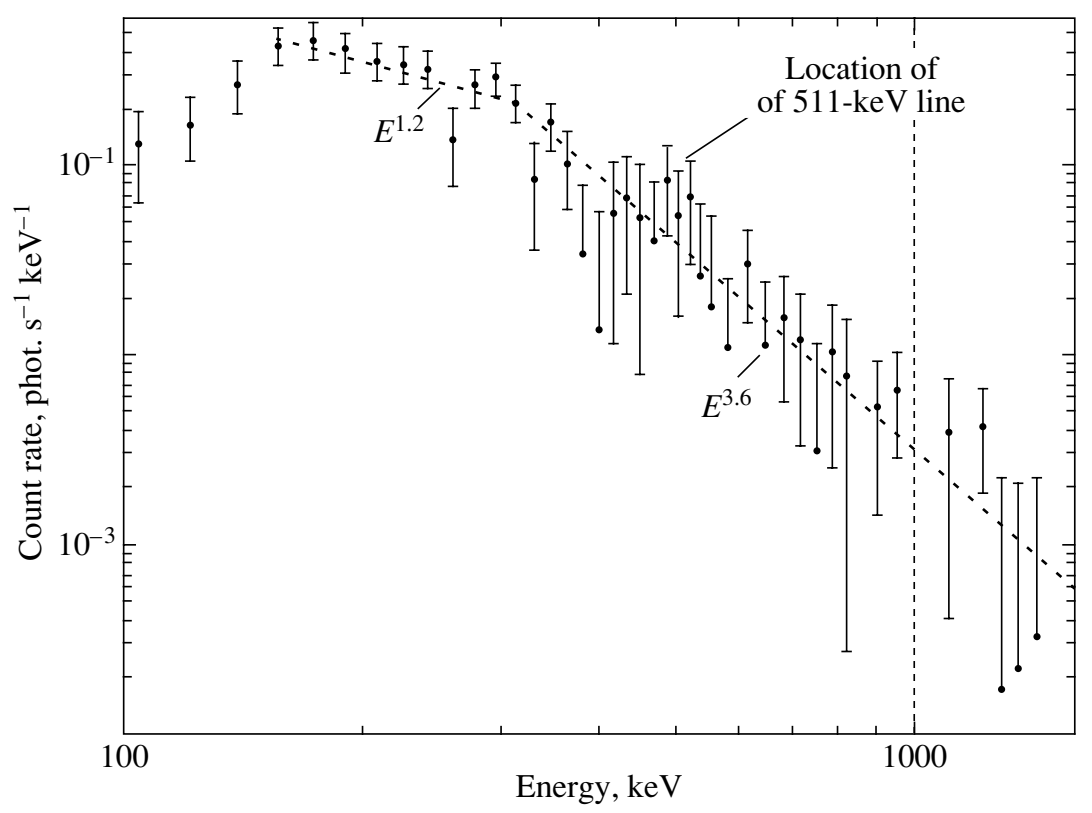

Fig. 9. The differential energy spectrum for the September 18, 2001 event.

THE IRIS FLARE SPECTROMETER

The instrument is designed to investigate the solar flare activity in the X-ray spectral range $2-200 \mathrm{keV}$. It has 12 energy channels with a temporal resolution of $2.5 \mathrm{~s}$ in patrol mode and 64 energy channels with a temporal resolution of $1 \mathrm{~s}$ and four channels with a $10 \mathrm{~ms}$ resolution in burst mode. As yet no spectral measurements have been carried out with such a temporal resolution in any experiment aimed at investigating the solar X-ray radiation. Observations 


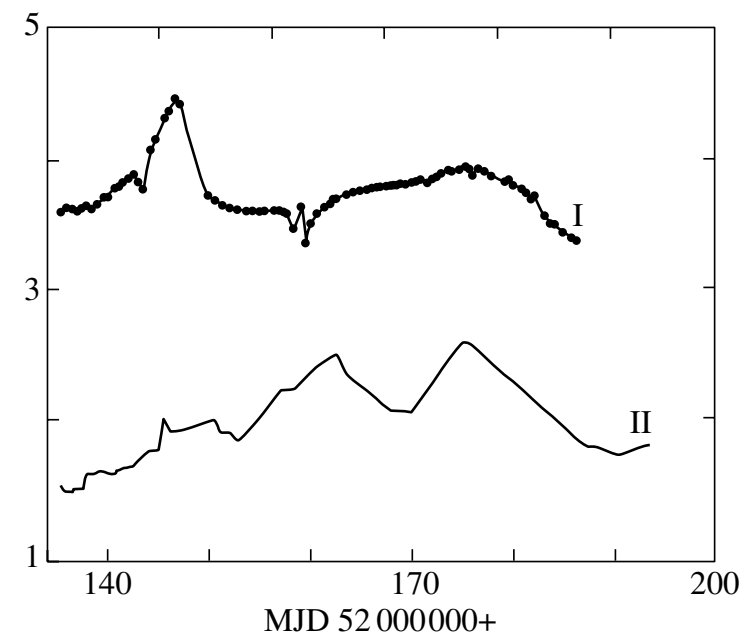

Fig. 10. The VUSS-L temporal record of a signal proportional to the solar UV flux (I) and the solar radio flux at a wavelength of $10.7 \mathrm{~cm}$ (II).

of the fine temporal structure in hard X-ray fluxes will provide direct information on the development of the energy release at the explosive phase of flares. Figure 7 shows an intensity record of hard X-ray radiation in four energy channels in the range 20$100 \mathrm{keV}$ with a high temporal resolution for the event of September 27, 2001, 18:26 UT.

\section{THE GELIKON GAMMA-RAY SPECTROMETER}

The instrument measures the temporal and spectral characteristics of the hard electromagnetic radiation from solar flares over a wide energy range, from $\mathrm{X}$-rays to gamma-rays $(10 \mathrm{keV}-10 \mathrm{MeV})$. It is used to monitor the radiation conditions and soft solar flares and to detect and record in detail hard $(E \gamma>50 \mathrm{keV})$ flares and gamma-ray bursts. Over the observing period from November 14 through December 14, 2001, the temporal profiles and spectra were measured for 134 solar flares. Most of them had soft spectra and were recorded in background mode with a temporal resolution of $1 \mathrm{~s}$ in eight adjacent energy bands from 15 to $200 \mathrm{keV}$. Figure 8 shows the temporal profile for the intense solar flare of November 23, 2001, detected in trigger mode in three energy bands with a high temporal resolution from 2 to $256 \mathrm{~ms}$.

\section{THE RPS-1 X-RAY SPECTROMETER}

The instrument is designed to investigate solar flares and their precursors in the X-ray range 3$30 \mathrm{keV}$. Over the period of measurements, the instrument calibration parameters were refined, the background conditions were mapped, and the experimen- tal data statistics was accumulated. No SOHO measurements are carried out in this energy range, while the SXS/Yohkoh results have not yet been published.

\section{THE ATS AMPLITUDE-TIME SPECTROMETER}

The instrument is designed to study the $\mathrm{X}$-ray and gamma-ray radiation from solar flares, in particular, to analyze solar-flare or gamma-ray burst events in the energy bands $3-30 \mathrm{keV}, 0.1-8.0 \mathrm{MeV}$, and 2.0$80.0 \mathrm{MeV}$. Over the observing period, several tens of candidates for solar-flare or gamma-ray burst events were recorded and their energy spectra were obtained for various phases of the event (before, during, and after the event). The duration of the candidate events ranges from 16 s to $3-5$ min. In Fig. 9, the differential energy spectrum for the event of September 18, 2001, was reconstructed from measurements. It exhibits a kink near energy $300 \mathrm{keV}$ and a possible spectral feature that can be identified with the $511-\mathrm{keV}$ annihilation line.

\section{THE SUVR-Sp-C SOLAR ULTRAVIOLET RADIOMETER}

The SUVR-Sp-C instrument measures the fluxes from the Sun as a star in several spectral bands, from 1 to $130 \mathrm{~nm}$. In contrast to $\mathrm{SOHO}$, it has a wider spectral range. Apart from the scientific objectives, the radiometer monitors one of the most important elements of cosmic weather-the geoeffective solar radiation. the SUVR obtained data on the intense X5.3B flare of August 25 at 16:23 UT at X-ray wavelengths $\lambda<12 \mathrm{~nm}$.

\section{THE VUSS-L ULTRAVIOLET SOLAR SPECTROPHOTOMETER}

The VUSS-L instrument carries out measurements in a band near $120 \mathrm{~nm}$ and is designed to investigate the solar ultraviolet radiation near the $L_{\alpha}$ resonance line. The measurements yield numerous data on the UV flux and intensity in the $L_{\alpha}$ line, which was tentatively estimated to be $\sim 10 \mathrm{erg} \mathrm{cm}^{-2} \mathrm{~s}^{-1}$. The VUSS-L temporal record of the UV flux is shown in Fig. 10.

\section{THE SCR INSTRUMENTATION TO STUDY SOLAR COSMIC RAYS}

The SCR scientific instrumentation consists of three instruments: SONG (gamma-ray and neutron spectrometer, principal investigator S.P. Ryumin), CRM (cosmic-ray monitor, principal investigator S.N. Kuznetsov), and CRS-3 (cosmic-radiation spectrometer, principal investigator A.F. Podorol'skii). 


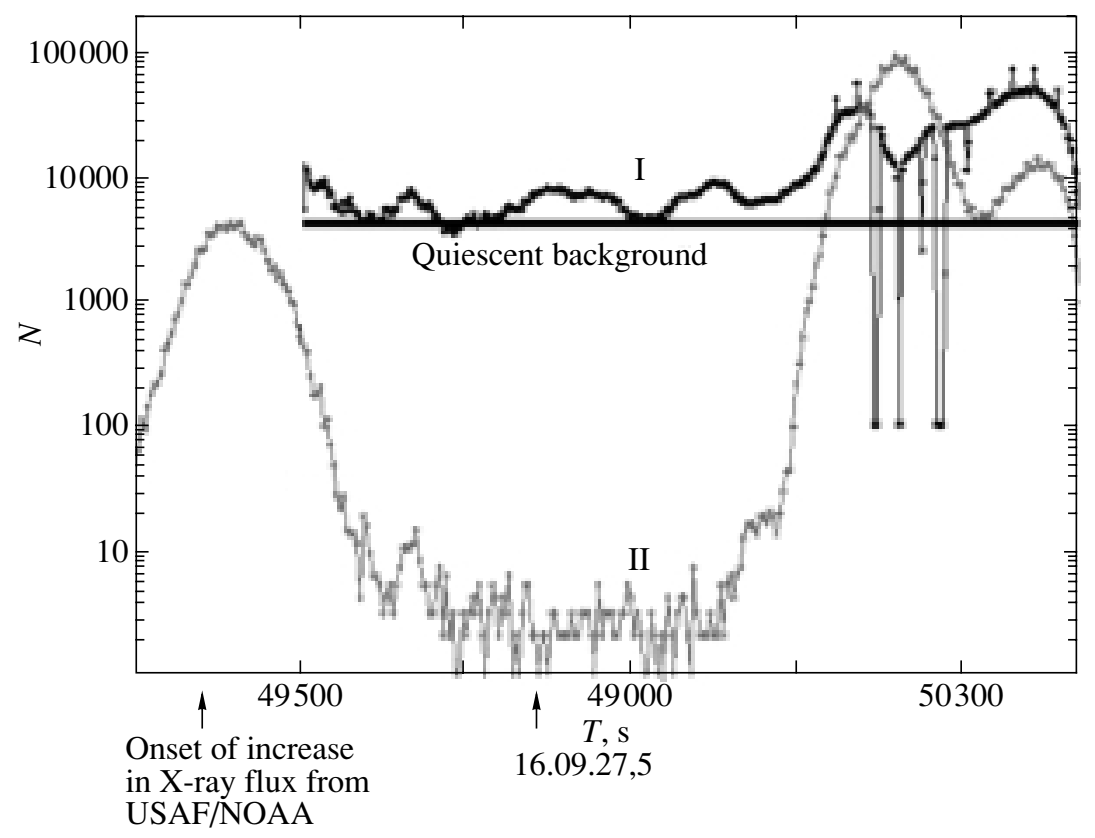

Fig. 11. The recording of gamma rays with energy 60-150 keV (I) and electrons with energy $0.3-0.6 \mathrm{MeV}$ (II) by the SCR complex in the November 4, 2001 event.

It is designed for comprehensive studies of solar cosmic rays. The SONG instrument records $\mathrm{X}$-ray and gamma-ray spectra in the energy range 0.03$100 \mathrm{MeV}$, detailed gamma-ray line spectra in the range $0.3-20 \mathrm{MeV}$, neutrons with energies $>20 \mathrm{MeV}$, and fluxes of charged cosmic-ray particles-protons with energies $>70 \mathrm{MeV}$ and electrons with energies $>50 \mathrm{MeV}$. The SRM instrument measures the fluxes and spectra of protons with energies $1-200 \mathrm{MeV}$ and electrons with energies $0.5-12 \mathrm{MeV}$. The CRS-3 measures the chemical composition and spectra of ions in the range $Z=1-10$ and in the energy range 1.5-20 MeV per nucleon for $\mathrm{He}$ and 4-40 $\mathrm{MeV}$ per nucleon for Ne. In comparison with the SOHO and Yohkoh instruments, the SONG spectrometer is capable of recording energetic (with energies up to $100 \mathrm{MeV}$ ) gamma-rays, which, in turn, makes it possible to observe gamma-rays from the decay of the $\pi^{0}$ mesons produced in interactions of highenergy protons. The CORONAS-F measurements show that the SONG background for recording solar neutrons is at least a factor of 5 to 7 lower than that of GRS (SMM) - the only instrument that recorded solar neutrons in the same energy range. Figure 11 shows the SONG recording of gamma-rays with energy 60-150 keV (I) and electrons with energy 0.3-0.6 MeV (II). The first increase in gammaray flux correlates with the electron flux and can be explained in part as electron bremsstrahlung. The two remaining increases represent solar flare radiation. Since the flare occurred in the western hemisphere, the recorded increase in relativistic particle flux began almost immediately.

\section{ACKNOWLEDGMENTS}

V.D. Kuznetsov and I.A. Zhitnik played a major role in designing the CORONAS-F complex. They also helped in writing this paper, for which we thank them. We are also grateful to T.V. Kazachevskaya, Yu.D. Kotov, G.E. Kocharov, S.N. Kuznetsov, N.I. Lebedev, E.P. Masetz, A.A. Nusinov, V.M. Pankov, A.F. Podorol'skii, S.P. Ryumin, and J. Silvestr, who provided the reduced data from the CORONASF satellite.

\section{REFERENCES}

1. A. Jiménez, P. L. Palle, T. Roca Cortes, and V. Domingo, Astron. Astrophys. 193, 298 (1988).

2. N. I. Lebedev, V. E. Oraevsky, Y. D. Zhugzda, et al., Astron. Astrophys. 296, L25 (1995).

3. V. N. Oraevsky, N. I. Lebedev, I. M. Kopaev, et al., oral communication (1999).

4. I. I. Sobelman, I. A. Zhitnik, and A. P. Ignat'ev, Pis'ma Astron. Zh. 22, 604 (1996) [Astron. Lett. 22, 539 (1996)].

5. The SOHO Mission: Scientific and Technical Aspects of the Instruments (ESA, Paris, 1988), ESA SP-1104.

6. I. Zhitnik, A. Ignatiev, V. Korneev, et al., Proc. SPIE 3406, 1 (1998).

Translated by V. Astakhov 